\title{
COVID-19: GEMFIBROZIL AS A POTENTIAL ADJUVANT IMMUNE MODULATING TREATMENT
}

\author{
Marciano Lee ${ }^{1}$, Elise Anderson ${ }^{1}$, Ali Farooq ${ }^{1}$, Mohammed Yasin ${ }^{1}$, Yazan Alkawaleet ${ }^{1}$, and \\ Frank Annie ${ }^{2,3}$ \\ ${ }^{1}$ Charleston Area Medical Center, Department of Cardiology \\ ${ }^{2}$ Department of Cardiology), CAMC Health Education and Research Institute \\ ${ }^{3} \mathrm{CAMC}$ Health Education and Research Institute
}

November 3, 2020

Marciano Lee $^{1}$, Elise Anderson ${ }^{1}$, Ali Farooq ${ }^{1}$, Mohammed Yasin ${ }^{1}$, Yazan Alkawaleet ${ }^{1}$, Frank Annie ${ }^{2}$

Charleston Area Medical Center, Department of Cardiology, 3200 MacCorkle Ave. SE,

Charleston, WV 25304

CAMC Health Education and Research Institute, 3200 MacCorkle Ave. SE,

Charleston, WV 25304

Corresponding:

Frank Annie MA; MPA; PhD

Research Scientist (Department of Cardiology)

CAMC Health Education and Research Institute

3200 MacCorkle Ave. SE,

Charleston, WV 25304

Cell $304-395-3830$

Phone 304-388-9921

Fax: 304-388-9921

Email: Frank.H.Annie@camc.org

Keywords:

COVID-19, GEMFIBROZIL, IMMUNE MODULATING TREATMENT

Abstract:

The current Covid-19 viral infection has been declared a pandemic causing significant global morbidity and mortality predominantly from the manifestation of severe acute respiratory syndrome (SARS). It has been designated as SARS-CoV 2. Epidemiological studies show predilection in patients with associated chronic medical conditions including those with cardiovascular disease. Exaggerated host immune response known as cytokine storm syndrome (CSS) leading to acute lung injury (ALI) and acute respiratory distress 
syndrome (ARDS) is the proposed mechanism for the development of SARS in Covid-19 infection. Gemfibrozil has proven benefits in patients with cardiovascular disease. More importantly, it has established anti-inflammatory, anti-oxidative, and anti-migratory properties. Gemfibrozil has shown survival benefits as immune modulation of ALI/ARDS from viral pathogens. This article reviews the potential of gemfibrozil as adjuntive immue modulating therapy for SARS associated with Covid-19 infected patients who have associated cardiovascular disease.

\section{Introduction:}

The severe acute respiratory syndrome (SARS) seen in Covid-19 infection share similarities with previous coronavirus epidemics, particularly SARS-CoV 1 and the Middle East Respiratory Syndrome (MERS-CoV). (1) It has been designated SARS-CoV 2. Acute lung injury (ALI) with subsequent development of acute respiratory distress syndrome (ARDS) from robust host hyper-immune response is the proposed pathophysiological mechanism. This so called "cytokine storm" may be independent of viral load and replication. (2) Thus, the rationale for adding immune modulating strategies on top of antiviral/antimicrobial therapies is warranted to prevent this complication and hopefully improve survival. The development of ALI/ARDS amplifies mortality in Covid-19 infection, more so in patients with associated chronic medical conditions including cardiovascular disease. (3)

Gemfibrozil, a drug discovered over 40 years ago, have beneficial effects and safety profile in both primary and secondary prevention of cardiovascular disease. (4) In addition to its lipid lowering ability, it has important immune modulating properties that inhibit pro-inflammatory factors involved in cytokine storms. (5) It is a peroxisome proliferators-activated receptor (PPAR) agonist known to reduce the release of inflammatory cytokines including interleukin-6 (IL-6), tumor necrosing factors (TNF) and interferon-gamma (IFN-g).

IL-6 elevation have been reported as an important marker of severity with Covid-19 infection. (3) Together with other markers for inflammation such as CRP, the degree of IL-6 elevation correlates with increased morbidity and mortality.

Gemfibrozil has shown survival benefits by attenuating ALI/ARDS development from viral etiologies. (6) Since gemfibrozil is currently indicated for patients with cardiovascular disease, its adjunctive use does not have to be repurposed for SARS associated with Covid-19 infection. Therefore, Gemfibrozil's potential as an immune modulating strategy to prevent ALI/ARDS cannot be ignored.

Immune Modulating Targets for Gemfibrozil in Covid-19 SARS pathogenesis:

Gemfibrozil has been shown to attenuate pro-inflammatory effects of disease states through its immunemodulating, anti-inflammatory and anti-migratory properties. (5). It belongs to the fibrate family of drugs used to lower plasma lipds and cholesterol. They are one of several known synthetic lignads acting as agonists to the PPARs. (7) Their action in turn decrease serum levels of TNF, IFN-g and IL-6. $(8,9)$

As an an anti-inflammatory, gemfibrozil reduces superoxide production and expression of nuclear factor $\mathrm{kB}$ (NF-kB). (10) Moreover, it inhibits astrocyte production of TNF, IL-1b, IL-6 and nitric oxide (NO) production. (11) Gemfibrozil also promotes the production of anti-inflammatory cytokine IL-4. (12) It reduces the production of C-reactive protein (CRP), TNF-a and IL-6 in peripheral blood mononuclear cells. It inhibits the production of cyclooxygenase-2 (COX-2) and prostaglandins. (13)

As an immune-modulating agent, gemfibrozil attenuates pro-inflammatory transcription factor activation. This includes IFN-g regulatory factor 1 (IRF-1)bidning to gamma activation site (GAS), nuclear factor$\mathrm{kB}(\mathrm{NF}-\mathrm{kB})$, activator protein $1(\mathrm{AP}-1)$, and $\mathrm{CCAT} /$ enhancer binding protein beta $(\mathrm{C} / \mathrm{EBPb})$. (5) It also promotes switching of $\mathrm{T}$ helper (Th) cells from the pro-inflammatory Th1 to anti-inflammatory Th2. Production of Th2-specific cytokines is amplified including IL-4, IL-5 and IL-10. (14)

As an anti-oxidant, gemfibrozil not only prevents low density lipoprotein (LDL) peroxidation but also acts on other signals of inflammation. Reactive oxygen species (ROS) have emerged as important signaling molecules during inflammatory conditions. Excessive ROS generation results in damage to most cellular components 
of the living organism. Gemfibrozil is known to inhibit ROS-mediated inflammation. (15) It has free radical scavenging ability as well. Its para-hydroxyl metabolite has been shown to reduce the burden of ROS. (16)

As an anti-migratory agent, gemfibrozil affects the expression of cell-surface adhesion molecules icluding intracellular adhesion molecule 1 (ICAM-1), VCAM-1 and selectins inhibiting trans endothelial migration of mononuclear cells and macrophages. (17) It also down-regulates the expression of chemokines including monocyte chemotactic protein 1 (MCP-1), macrophage inflammatory protein 1 (MIP-1), monokines induced by IFN-g (MIG) and IL-8. The expression of IL-8 induces inflammation, monocyte/macrophage recruitment, angiogenesis and vascular smooth muscle migration. Thus, gemfibrozil can function as an anti-migratory factor by down-regulating the expression of different soluble chemokines and inhibit the expression of different cell surface proteins and selectins.

Discussion:

Over the years, viral pathogens including Influenzas and Coronaviruses cause ALI/ARDS from excessive release of pro-inflammatory cytokines and chemokines described in cytokine storm syndromes. They induce much higher transcription and up regulation of TNF genes including TNF related aptoptosis-inducing lignad and TNF-mRNA in human monocyte-derived macrophages. (18) This macrophage activation syndrome (MAS) seen with the current SARS-CoV 2 may be a dysregulated host response to Covid-19 viral induced immunosuppression and lymphopenia leading to unintended ALI/ARDS consequences at the site of infection. (19) The development of ALI/ARDS and the need for ventilator support portends to increased mortality rates. Markers of inflammation including CRP and IL-6 have been used as surrogates for MAS, and their degree of elevation have correlated well with disease severity. (3)

Experiments with SARS-CoV indicate that MAS induced cytokine storms result more from amplification of gene related inflammatory signals regardless of viral load. In one model, immune modulation of receptor sites or alteration of recruitment signals resulted in dramatic improvement in host survival with viral load remaining constant. (20) This suggests that immune modulating therapy may have as important a role in addition to antiviral therapy in altering the course of Covid-19 infection. Reports of clinical improvement have been reported with the adjuvant use of IL-6 antagonists including tocilizumab. However, cost and repurposing use of these medications may be limiting factors.

Gemfibrozil has been in clinical use for patients with cardiovascular disease and has achieved generic status. In addition, it has vital immune modulating properties against cytokine storms that mitigate the development of ALI/ARDS from both viral and nonviral pathogens. (21). It not only reduces the levels of IL-6, TNF and IFN-g. But it has also been shown to attneuate gene recruiting signals of inflammation utlizing human equivalent dosing. In one animal study, the adjuvant use of gemfibrozil resulted in enhanced host survival that developed ALI/ARDS from viral pathogens. (6) Enhanced survival is seen even after exposure to the virus. This implies that gemfibrozil has the potential to be a treatment rather than a preventive therapy in human disease.

Therefore, the potential of gemfibrozil as adjunct immune modulating therapy in conjunction with antiviral treatment for SARS associated with Covid-19 infection in patients with associated cardiovascular disease is emphasized.

References:

1. Petrosillo N, Vicenconte G, et al. Covid-19, SARS and MERS: are they closely related? JCMI. 2020

2. Smits S, Lang A, et al. Exacerbated inane host response to SARS-CoV in aged non-human primates. PLos Patholog. 2010

3. Zhou F, Du R, et al. Clinical course and risk factors for mortality of adult inpatients with Covid-19 in Wuhan, China: a retrospective cohort study. Lancet. 2020. 395

4. Rubins HB, Robins SJ, et al. Gemfibrozil for the secondary prevention of coronary aretery disease inmen with low levels of high-density lipoprotein cholesterol. Veterans Affairs high-density lipoprotein cholesterol 
intervention trial group. N Engl J Med. 1999. 341: 410-418

5. Roy A, Pahan K, et al. Gemfibrozil, stretching the arms beyond lipid lowering. Immunopharmacol Immunotoxicol. 2009. 31: 339-351

6. Budd A, Alleva L, et al. Increased survival after gemfibrozil treatment of severe mouse influenza. Antimicrobial Agents and Chemotherapy. 2007. 2965-2968

7. Berger J, Moler DE. The mechanisms of action of PPARs. Annu Rev Med. 53: 409-435

8. Madej A, Okopien B, et al. Effects of fenofibrate on plasma cytokine concentrations in patients with atherosclerosis and hyperlipoproteinemia IIb. Int J Clin Pharmacol. 36: 345-349

9. Staels B, Koenig W, et al. Activation of human oartic smooth muscle cells is inhibited by PPARa. Nature. 303: $790-793$

10. Calkin AC, Cooper KA, et al. Gemfibrozil decreases atherosclerosis in experimental diabetes in association with a reduction in oxidative stress and inflammation. Diabetologia. 49: 766-774

11. Xu J, Chavis JA, et al. Gemfibrozil reduces release of tumor necrosis factor-alpha in peripheral blood mononuclear cells from healthy subjects and patients with coronary heart disease. Clin Chem Acta. 332: $61-67$

12. Lovette-Racke AE, Hussain RZ, et al. Peroxisome proliferator-activated receptor-alpha agonists as therapy for autoimmune diesease. J Immunol. 172: 5790-5798

13. neve BP, Frutchart JC, et al. Role of PPAR in atherosclerosis. Biochem Pharmacol. 2000. 60: 1245-1250

14. Heyworth PG, Bohl BP, et al. Rac translocates independently of the neutrophil NADPH oxidase components p47phox and p67phox. Evidence for its ineteraction with flavocytochrome b558. J Biol Chem. 1994. 269: 30749-30752

15. Aviram M, Rosenblat M, et al. Atorvastatin and gemfibrozil metabolites, but not the parent drugs, are potent antioxidants against lipoprotein oxidation. Ahterosclerosis. 1998. 138: 271-280

16. Dasgupta S, Roy A, et al. Gemfibrozil ameliorates relapsing-remitting experimental autoimmune encephalomyelitis of peroxisome proliferator-acivated receptor-alpha. Molecular Pharmacol. 2007. 72: 934-946

17. Zhou JF, Law HK, et al. Functional tumor necrosis factor-related apoptosis-inducing ligand production by avian flu influenza virus-infected macrophages. J Infect Dis. 193: 945-953

18. Xu J, Shi I, et al. Pathological findings of Covid-19 associated with acute respiratory distress syndrome. Lancet. 2020. Epub ahead of print

19. McGonagle D, Sharif K, et al. The role of cytokines including interleukin-6 in Covid-19 induced pneumonia and macrophage activation syndrome-like disease. J AutRev. 2020. 102537

20. Channappanavar AR, Fehr R, et al. Dysregulated type 1 interferon and inflammatory monocytesmacrophage responses cause lethal pneumonia in SARS-CoV infected mice. Cell Host Microbe. 2016.19: 181-193

21. Kim YS, Kim JK, et al. The peroxisome proliferator-activated receptor-alpha agonist gemfibrozil promotes defense against mycobacterium abcessus infections. Cells. 2020. 9: 648 